OPEN ACCESS

Edited by:

Katja Teerds,

Wageningen University \& Research,

Netherlands

Reviewed by:

Rajprasad Loganathan,

Johns Hopkins University School of

Medicine, United States

Andrew Swan,

University of Windsor, Canada

*Correspondence:

Evdokia Dimitriadis

eva.dimitriadis@unimelb.edu.au

Specialty section:

This article was submitted to

Cell Growth and Division,

a section of the journal

Frontiers in Cell and Developmental

Biology

Received: 23 July 2020

Accepted: 24 August 2020

Published: 22 September 2020

Citation:

Zhou W and Dimitriadis E (2020)

Secreted MicroRNA to Predict

Embryo Implantation Outcome: From

Research to Clinical Diagnostic

Application

Front. Cell Dev. Biol. 8:586510. doi: $10.3389 /$ fcell.2020.586510

\section{Secreted MicroRNA to Predict Embryo Implantation Outcome: From Research to Clinical Diagnostic Application}

\author{
Wei Zhou ${ }^{1,2}$ and Evdokia Dimitriadis ${ }^{1,2 *}$ \\ ${ }^{1}$ Department of Obstetrics and Gynaecology, The University of Melbourne, Parkville, VIC, Australia, ${ }^{2}$ Gynaecology Research
} Centre, The Royal Women's Hospital, Parkville, VIC, Australia

Embryo implantation failure is considered a leading cause of infertility and a significant bottleneck for in vitro fertilization (IVF) treatment. Confirmed factors that lead to implantation failure involve unhealthy embryos, unreceptive endometrium, and asynchronous development and communication between the two. The quality of embryos is further dependent on sperm parameters, oocyte quality, and early embryo development after fertilization. The extensive involvement of such different factors contributes to the variability of implantation potential across different menstrual cycles. An ideal approach to predict the implantation outcome should not compromise embryo implantation. The use of clinical material, including follicular fluid, cumulus cells, sperm, seminal exosomes, spent blastocyst culture medium, blood, and uterine fluid, that can be collected relatively non-invasively without compromising embryo implantation in a transfer cycle opens new perspectives for the diagnosis of embryo implantation potential. Compositional comparison of these samples between fertile women and women or couples with implantation failure has identified both quantitative and qualitative differences in the expression of microRNAs (miRs) that hold diagnostic potential for implantation failure. Here, we review current findings of secreted miRs that have been identified to potentially be useful in predicting implantation outcome using material that can be collected relatively non-invasively. Developing non-invasive biomarkers of implantation potential would have a major impact on implantation failure and infertility.

Keywords: embryo implantation, non-invasive prediction, microRNAs, male factor, spent blastocyst culture medium, blood, uterine fluid, oocyte quality

\section{INTRODUCTION}

Infertility affects a staggering one in six couples worldwide (Wilcox et al., 1988) and can be a devastating condition for couples, with the failure to conceive recognized as a leading cause of psychological distress, depression, low self-esteem, and domestic violence (Chachamovich et al., 2010; Cui, 2010). A major contributor to infertility is the failure of blastocysts to implant, accounting for $>50 \%$ of all failed pregnancies (Craciunas et al., 2019). While in vitro fertilization (IVF) has increasingly assisted couples to conceive, success rates have stagnated as still, $\sim 50 \%$ 
of good quality blastocysts fail to implant (Gardner and Balaban, 2016). Implantation is a highly complex biological process that requires the coordination between a healthy embryo and a receptive endometrium. The process is initiated via fertilization of a healthy oocyte, which occurs in the Fallopian tube. During fertilization, the female reproductive tract serves as a natural selection system to guarantee that the best quality sperm reaches and fertilizes the oocyte (Ralt et al., 1991). Once fertilized, the zygote travels through the Fallopian tube and develops to the morula stage when it reaches the uterine cavity. The morula stage embryo continues to develop to the blastocyst stage in the uterine cavity before implantation (Norwitz et al., 2001). This can take up to $72 \mathrm{~h}$ within which time the embryo and the endometrium communicate via secreted and cell surface factors to prepare for the initial adhesion and attachment (Ashary et al., 2018). Once the outer layer of the embryo, namely, the trophectoderm firmly attaches to the endometrial luminal epithelium, it initiates implantation. Failure of firm adhesion leads to implantation failure.

Successful implantation is based on the cumulative success of the above events. Implantation can be affected by many factors including sperm and oocyte quality, early development of the embryo, the endometrium, and the reciprocal communication between blastocysts and endometrium. During an IVF clinical setting, embryo quality is generally scored via assessment of morphology, expansion and hatching, development of inner cell mass, and the formation of the trophectoderm layer (Giorgetti et al., 1995; Gardner et al., 2000). The transfer of embryos graded as good or "transferable" can improve implantation and pregnancy outcome (Giorgetti et al., 1995; Gardner et al., 2000). However, these morphological criteria do not necessarily correlate with implantation potential. Embryos with similar morphologically good scores assessed to be of transferable quality from aged women ( $>38$ years) have a significantly lower pregnancy rate compared to those of younger women ( $<38$ years) (Giorgetti et al., 1995). It is estimated that overall, $50 \%$ of good quality embryos fail to implant (Gardner and Balaban, 2016). In addition to scores based on morphology, preimplantation genetic testing is also used in some IVF clinics. This testing requires the collection of trophectoderm cells to assess the ploidy of blastocysts and can reveal one of the many characters that may affect implantation. Another clinical approach to improve implantation success is via the assessment of endometrial receptivity. A current clinical test, called endometrial receptivity array, is used to evaluate whether the endometrium is in phase or receptive (Díaz-Gimeno et al., 2011). However, this method is invasive as it requires an endometrial biopsy and does not diagnose a disrupted or dysregulated endometrium, and while promising, there is still a need to develop non-invasive methods to recognize a disrupted endometrium (Díaz-Gimeno et al., 2011). In addition to the endometrium, sperm quality also can affect implantation. Current clinical analysis of sperm quality relies on the basic assessments of sperm morphology and motility, which do not necessarily reflect their capability in facilitating embryo development and implantation.

Despite the available tests, abnormalities in sperm, oocytes, disrupted endometrium, and embryo-endometrial interactions that contribute to implantation failure are not able to be effectively determined, and implantation failure remains a significant bottleneck for IVF treatment. To improve this, emerging work focuses on assessing biomarkers in samples that can be collected relatively non-invasively and examining whether they reflect the implantation potential. While many different classes of potential biomarkers have been proposed, microRNAs (miRs) stand out as promising biomarkers to determine the quality of sperm, oocytes, embryos, and endometrium that could be used to predict implantation outcome. miRs are small non-coding RNAs that regulate gene expression and protein production (Bushati and Cohen, 2007). Secreted or extracellular miRs are highly stable in body fluids, reflect disease states, and are easily detectable in a short time frame making them highly suitable for biomarker detection (Cuman et al., 2015). Emerging evidence strongly suggests that miRs regulate human embryo implantation (Paul et al., 2019). Recent studies support their use as non-invasive biomarkers for sperm, oocyte, and blastocyst quality, endometrial receptivity, and blastocystendometrial interactions (Cuman et al., 2015; Machtinger et al., 2017; Li et al., 2019; Abu-Halima et al., 2020). This review aims to discuss the use of miRs for screening blastocyst quality and implantation potential, focusing on using human samples that can be collected relatively non-invasively.

\section{LABORATORY IDENTIFICATION OF MIRS WITH TRANSLATIONAL POTENTIAL FOR IMPLANTATION PREDICTION}

A standard IVF treatment broadly requires egg retrieval, sperm collection, IVF, and embryo culture before transfer. The collection of cumulus cells and follicular fluid is possible during egg retrieval without affecting IVF (Figure 1). Analysis of gene and miR expression in follicular fluid and cumulus cells indicates oocyte and embryo quality, thus the implantation potential from an embryo's perspective (Hamel et al., 2008; Fu et al., 2018). Embryos are generally cultured up to 56 days to reach the blastocyst stage before transfer (Figure 1). Embryos secrete specific profiles of miRs that may reflect their quality and implantation potential (Kropp et al., 2014; Capalbo et al., 2016). The endometrial epithelium secretes factors into the uterine cavity to regulate implantation and uterine fluid, or uterine lavage washings can potentially be used to detect miRs as biomarkers for the prediction of receptivity and implantation (Boomsma et al., 2009). Blood contains extracellular miRs with expression levels of some miRs positively correlating with endometrial levels and can potentially indicate whether the endometrium is dysregulated or receptive (Kresowik et al., 2014). Abnormalities in sperm contribute to blastocyst development and quality (Yuan et al., 2016), which could impact implantation. It has been shown in mice that sperm relays epigenetic information to the oocyte during fertilization and influences pre-implantation embryo and offspring development (Sharma et al., 2016). The development of non-invasive biomarkers has driven extensive research in 


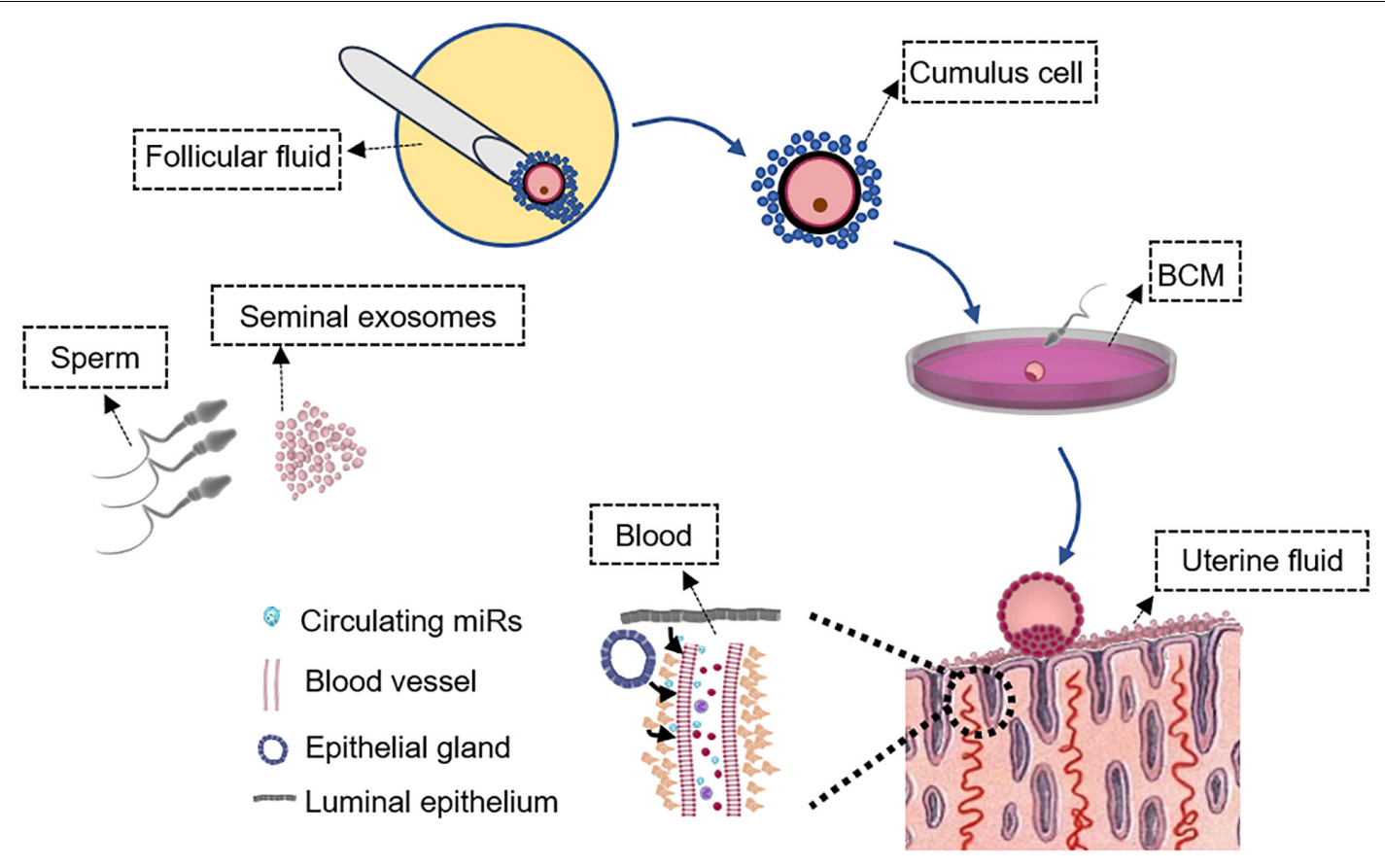

FIGURE 1 | Schematic of samples that can be collected non-invasively during in vitro fertilization (IVF) treatment. Characterization of the seminal exosome and sperm microRNA (miR) profiles could determine sperm quality to predict the potential of embryo development and IVF outcome. Analysis of miR expression in follicular fluid and cumulus cells may indicate oocyte and embryo quality, and implantation potential from an embryo's perspective. miR profiles in BCM also likely reflect embryo quality that may not be distinguished by morphology-based assessment. Endometrial cells release miRs in the blood and the expression levels of at least some endometrial miRs are reflected in the blood. Assessment of circulating miRs in the blood may predict endometrial receptivity and implantation outcome. Uterine fluid miR levels reflect "local" endometrial miR secretion and can be collected without compromising embryo transfer to provide information on endometrial receptivity. These samples are outlined. BCM, blastocyst culture medium.

this area with an overall aim to improve the success rate of implantation and IVF treatment.

\section{CUMULUS CELLS AND FOLLICULAR FLUID}

Cumulus cells are implicated in oocyte development and competence (Huang and Wells, 2010). In addition to interacting directly with the oocyte to facilitate maturation, cumulus cells are also bathed within the same follicular environment during oocyte maturation, thus may retain a footprint to reflect its quality and potential to form a viable embryo (Figure 1; Patrizio et al., 2007). It has been proven that cumulous cells are useful for non-invasive diagnosis of oocyte quality (Hamel et al., 2008; Devjak et al., 2016). Next-generation sequencing on human cumulus cells has revealed that miRs represent the major small RNA type, constituting as much as $71 \%$ of the total small RNAs (Xu et al., 2015). As a way of interaction, it has been shown that bovine cumulus cells and oocytes reciprocally affect the abundance of miRs in each cellular compartment (Abd El Naby, 2012), and these miRs readily control gene expression with extensive downstream functional implications. Gene expression studies on human cumulus cells have revealed transcripts that may be involved in oocyte maturation, implantation, and pregnancy with their regulatory miRs just beginning to be realized (Gasca et al., 2007; Hamel et al., 2008). During IVF treatment, cumulus cells are retrieved while still firmly attached to each oocyte, and as such, their collection can be sourced from individual oocytes and, thus provide an indication of the developmental potential for individual oocytes.

Follicular fluid is also collected during oocyte retrieval; however, unlike cumulus cells, follicular fluid normally collected during IVF stimulation is a pool from several oocytes, rather than a single oocyte to avoid multiple vaginal punctures. Despite this limitation, one study using pooled follicular fluid from individual patients has identified differences in miR expression between groups with different pregnancy outcomes (Scalici et al., 2016). This study screened five miRs and identified that hsa-miR-29a expression in the follicular fluid could predict pregnancy outcome with a specificity of $53.5 \%$ and has a higher discrimination power compared to prediction using embryo morphology scores (Scalici et al., 2016). Another two investigations collected follicular fluid from a single follicle and used microarrays to screen miRs that were able to predict the difference between good and bad quality blastocysts. Although hsa-miR-663b has been identified as a common miR that is inversely related to good quality blastocysts (Machtinger et al., 2017; Fu et al., 2018), the blastocyst quality discrimination method used in the two studies is based on routine morphological assessment. Therefore, the use of hsa-miR-663b as a marker of 
good quality blastocysts can similarly be determined by routine morphological assessment and likely provides limited application potential to predict embryo implantation outcome. In a clinical IVF setting, generally, multiple oocytes are retrieved at one time, and it is likely that they differ in quality and potential to develop into viable blastocysts. This may limit the extensive application of follicular fluid as it cannot be used to evaluate miRs released by individual oocytes.

\section{SEMINAL PLASMA AND SPERM}

Defective sperm function is widely acknowledged as a major contributor to infertility. Under physiological conditions, the sperm acquires functional competence during their transit through the epididymis and female reproductive tract. Both biophysical and biochemical changes occur along this journey, eventually culminating in the ability of sperm to undergo an acrosome reaction, recognize the oocyte, and contribute to embryo development (Zhou et al., 2018). A number of recent studies in mice have provided evidence that uptake of miRs from the epididymal luminal environment endows the sperm with the capability to contribute to the early embryonic development and, thus, implantation upon delivery to the oocyte (Yuan et al., 2016; Conine et al., 2018, 2019). miR profile comparisons between mouse caput and cauda sperm have identified $27 \mathrm{miRs}$ that are specifically enriched in cauda sperm, compared to caput sperm (Nixon et al., 2015; Sharma et al., 2018). Microinjection of cauda sperm-enriched miRs into caput-derived embryos rescue gene expression defects before implantation in mice (Conine et al., 2019). Further investigation has identified an epididymosomedependent mechanism for the selective delivery of miRs into the sperm during their transit in the epididymis in mice (Reilly et al., 2016; Trigg et al., 2019; Zhou et al., 2019). In humans, differences in miR expression profiles have been recorded in both seminal plasma and sperm relative to different embryo qualities and pregnancy outcomes (Mokánszki et al., 2019; Abu-Halima et al., 2020; Xu et al., 2020). miR sequencing analysis on sperm samples grouped according to different embryo qualities has identified higher expression levels of hsa-miR-191 in the sperm group with better embryo developmental outcome (Xu et al., 2020). hsa-miR-19b-3p has a lower expression in sperm that is associated with a successful pregnancy outcome (Abu-Halima et al., 2020). Another recent study selected 11 spermatogenesis-related miRs and revealed that hsa-let-7a, hsamiR-7-1-3p, hsa-miR-141, hsa-miR-200a, and hsa-miR-429 were significantly elevated, while hsa-miR-15b, hsa-miR-34b, and hsa-miR-122 were significantly downregulated in both seminal plasma and sperm of infertile male patients with impaired sperm production, compared to males with normal fertility (Mokánszki et al., 2019). Seminal plasma has been identified with an enriched population of epididymosome-like vesicles, namely, seminal exosomes (Vojtech et al., 2014). A limitation of using seminal exosomes is that they represent a mixed population of extracellular vesicles originating not only from the epididymis but also from the prostate and seminal vesicles (Rolland et al., 2013), Whether miR profiles in these vesicles correlate with sperm quality requires investigation. Nevertheless, seminal exosomes have been implicated in the transfer of cargo to sperm, which promotes their motility, ability to capacitate, and complete acrosomal exocytosis, therefore, affecting sperm quality (Tompkins et al., 2015). In addition, exosomes isolated from seminal plasma can modulate the immune response and gene expression changes in the female reproductive tract (Robertson and Sharkey, 2016; Bai et al., 2018), which eventually facilitate implantation and pregnancy in humans. Such functions are at least mediated via seminal exosome-carried miRs (Machtinger et al., 2016), and like epididymosomes, seminal exosomes carry distinctive profiles of miRs (Vojtech et al., 2014). Improved characterization of the seminal plasma and sperm miR profiles could not only be beneficial in terms of uncovering the causative basis of male gamete dysfunction but also for the provision of urgently needed biomarkers of sperm quality to reliably predict the outcome of IVF treatments.

\section{SPENT BLASTOCYST CULTURE MEDIUM}

In an IVF setting, a fertilized oocyte is generally cultured in vitro for up to 5-6 days to the blastocyst stage before transfer. Spent culture media can be collected during media change without affecting embryo quality. It has been demonstrated that over 96\% of miRs present in the spent culture media originate from the trophectoderm and can be consistently detected after blastulation under IVF culture conditions (Capalbo et al., 2016). It is tempting to speculate that blastocyst-secreted miRs participate in the regulation of trophectoderm-endometrial luminal epithelial interactions therefore implantation. In keeping with this notion, it has been identified that embryos with different implantation outcomes (implanted versus non-implanted) secrete different profiles of miR into the culture medium (Cuman et al., 2015; Borges et al., 2016; Capalbo et al., 2016). Increased expression of hsa-miR-142-3p and decreased expression of hsa-miR-20a and hsa-miR-30c have been identified in non-implanted blastocyst culture medium (BCM), compared to implanted BCM (Table 1; Borges et al., 2016; Capalbo et al., 2016). Further, microarray screens have identified a list of miRs exclusively detected in either implanted or non-implanted BCM (Table 1; Cuman et al., 2015; Capalbo et al., 2016). miR profiles in the BCM also likely reflect embryo quality and overall IVF outcome, as summarized in Table 1 (McCallie et al., 2010; Rosenbluth et al., 2014; AbuHalima et al., 2020).

A previous study has proposed that while the pre-implantation embryo is in the uterine cavity, it packages regulatory miRs into extracellular vesicles (Ashary et al., 2018). They further propose that the packaged miRs are taken up by the endometrial luminal epithelial cells and alter their function to prepare for implantation. Incubation of primary human endometrial epithelial cells (HEECs) with BCM collected from embryos that were implanted increases their adhesive capacity to trophoblast cell line-formed spheroids (Cuman et al., 2013). Other notable examples include hsa-miR-661, which is exclusively secreted by blastocysts that fail to implant (Cuman et al., 2015). 
TABLE 1 | Identified microRNAs (miRs) in blastocyst culture medium (BCM) with diagnostic potential.

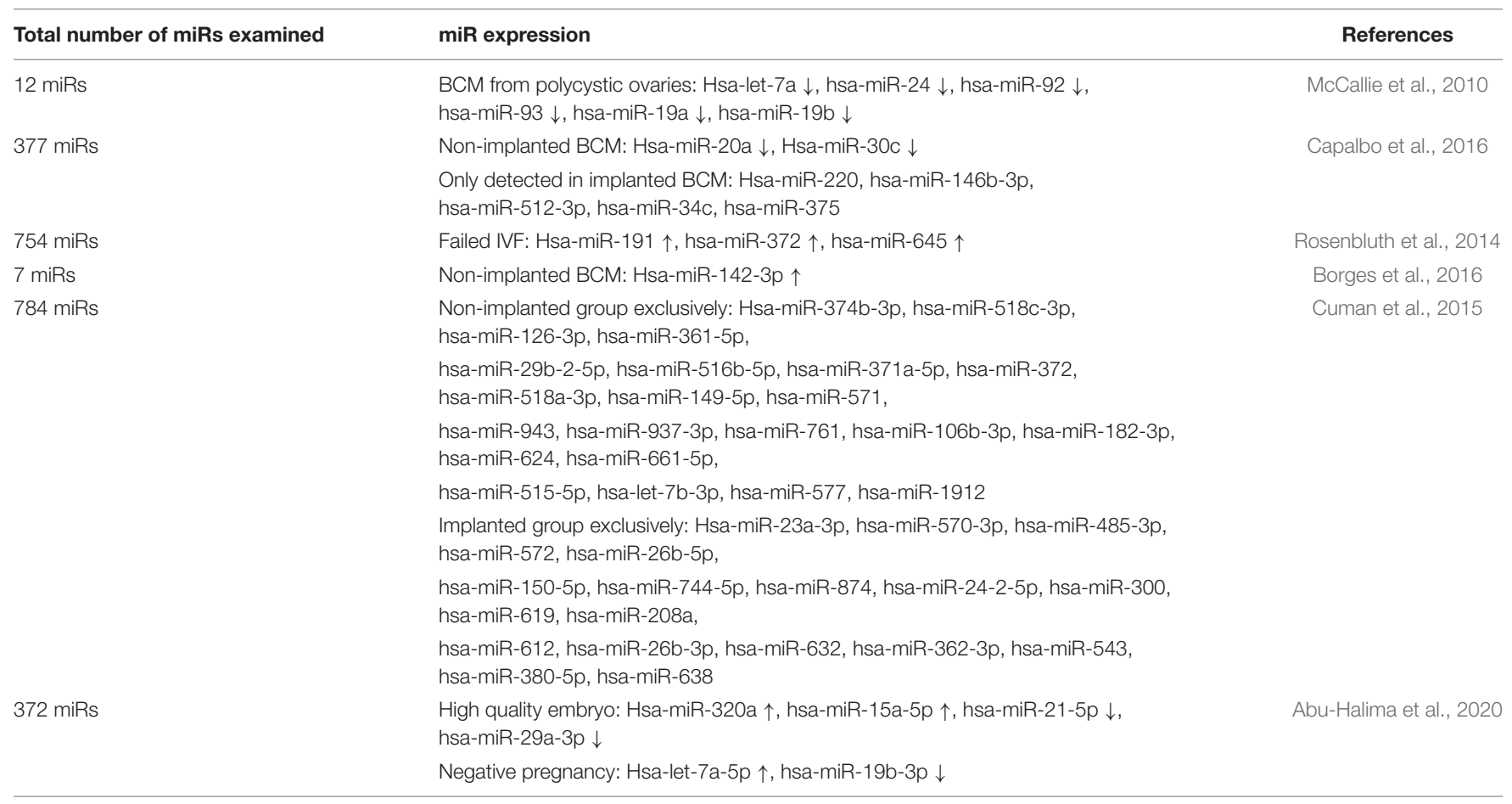

Secreted hsa-miR-661 from non-implanted BCM is taken up by HEECs and reduces their adhesion to trophoblast cellformed spheroids (Cuman et al., 2015). A recent study also demonstrates that incubation of HEECs with BCM from embryos that implanted, compared to embryos that did not implant during IVF, leads to a substantial change in the expression of long non-coding RNAs in the HEECs (Takamura et al., 2019). PTENP1 is one of the most decreased long non-coding RNAs in HEECs after being treated with BCM from embryos that fail to implant (Takamura et al., 2019). Functionally, knockdown of PTENP1 impairs HEEC adhesion via a miR-dependent mechanism to downregulate gene targets essential for receptivity (Takamura et al., 2019).

The implanted and non-implanted embryos from which BCM was collected had an indistinguishable morphology based on currently available assessment of embryo quality. Thus, miRs in the BCM may serve as promising non-invasive biomarkers to improve the diagnostic accuracy of embryo quality and implantation potential. An obvious challenge to achieve this is to determine which cohorts of miRs are present in BCM samples that correlate with implantation outcome, in particular, regardless of embryo culture conditions. Although some miRs such as hsa-miR-19b-3p and hsa-miR-372 have been identified in at least two independent studies (Table 1), the comparison of secreted miRs in BCM with different implantation outcomes has demonstrated a generally inconsistent result among different studies (McCallie et al., 2010; Rosenbluth et al., 2014; Cuman et al., 2015; Borges et al., 2016; Capalbo et al., 2016; Abu-Halima et al., 2020). Contributing factors to this inconsistency include diverse embryo culture conditions in different IVF clinics, unstandardized protocols, manual effects on RNA isolation, and
miR detection (Belandres et al., 2019). In addition, an obvious confounder of associating miRs in BCM with failed implantation outcome is the potential effects of the endometrium. A failed implantation group from which BCM was collected could be due to poor embryo quality, dysregulated endometrium, or altered receptivity window. The communication between an embryo and endometrium remains a "black box," and it is perhaps notable that not all secreted miRs are taken up by endometrial luminal epithelial cells to regulate implantation. For future diagnostic purposes, it is necessary to identify which miRs are taken up by endometrium and their actions on the endometrium. A panel of miRs will likely to be included, with the functional consequence of each individual $\mathrm{miR}$ on implantation being confirmed in ideally both humans (in vitro) and preclinical animal models (in vivo). Detailed functional studies have only covered a small proportion of miRs identified so far (Table 2; Chu et al., 2015; Cuman et al., 2015; Kang et al., 2015; Kottawatta et al., 2015; Vilella et al., 2015; Zhang et al., 2015; Cai et al., 2016; Chen et al., 2016; Zheng et al., 2017; Sirohi et al., 2018; Winship et al., 2018; Balaguer et al., 2019; Griffiths et al., 2019; Takamura et al., 2019).

\section{BLOOD}

MicroRNAs are also readily secreted into the blood. Circulating miRs are packaged in membrane-bound vesicles, attached to high-density lipoproteins or bound to RNA-binding proteins, which endow them with striking stability in the blood (Schwarzenbach et al., 2014). The human endometrium features a rich blood supply with the responsibility to 
TABLE 2 | miRs that regulate embryo implantation.

\begin{tabular}{|c|c|c|c|c|}
\hline miR & Phenotype & Target (s) & $\begin{array}{l}\text { Affect implantation in } \\
\text { animal model }\end{array}$ & References \\
\hline Hsa-miR-200c & $\begin{array}{l}\text { Overexpression of miR-200c in RL95-2 and Ishikawa cells } \\
\text { impair receptive ability in vitro }\end{array}$ & FUT4 $\downarrow$ & Yes (mouse) & Zheng et al., 2017 \\
\hline \multirow[t]{2}{*}{ Hsa-miR-661 } & $\begin{array}{l}\text { Secreted by non-implanted embryo and transferred to } \\
\text { HEECs to affect their adhesive capacity in vitro }\end{array}$ & PVRL1 $\downarrow$ & Not available & Cuman et al., 2015 \\
\hline & $\begin{array}{l}\text { Overexpression of miR-661 in HEECs in vitro impairs } \\
\text { adhesion }\end{array}$ & MDM2 2 & Not available & Winship et al., 2018 \\
\hline Hsa-miR-181a & $\begin{array}{l}\text { Inhibition of miR-181a compromises human endometrial } \\
\text { stromal cell decidualization in vitro }\end{array}$ & 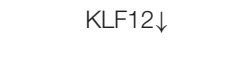 & Yes (mouse) & Chu et al., 2015; Zhang et al., 2015 \\
\hline Hsa-miR-212 & $\begin{array}{l}\text { hCG stimulates miR- } 212 \text { expression in Ishikawa cells to } \\
\text { favor spheroid adhesion in vitro }\end{array}$ & OLFM1 $\downarrow$, CTBP $1 \downarrow$ & Not available & Kottawatta et al., 2015 \\
\hline Hsa-miR-145 & $\begin{array}{l}\text { Overexpression of miR-145 in Ishikawa cells affects mouse } \\
\text { embryo attachment in vitro }\end{array}$ & $\mid G F 1 R \downarrow$ & Not available & Kang et al., 2015 \\
\hline \multirow[t]{2}{*}{ Hsa-miR-30d } & $\begin{array}{l}\text { Human endometrial secreted miR-30d is taken up by } \\
\text { mouse pre-implantation embryo and increases embryo } \\
\text { adhesion in vitro }\end{array}$ & $\operatorname{ltg} b 3 \uparrow, \operatorname{Itg} a 7 \uparrow, C d h 5 \uparrow$ & Yes (Mouse) & Vilella et al., 2015; Balaguer et al., 2019 \\
\hline & $\begin{array}{l}\text { Overexpression of miR-30d in Ishikawa cells facilitates } \\
\text { adhesion in vitro }\end{array}$ & $S N A / 1 \downarrow$ & Yes (Mouse) & Cai et al., 2016; Balaguer et al., 2019 \\
\hline Hsa-miR-125b & $\begin{array}{l}\text { Overexpression of miR-125b in HEECs inhibits cell } \\
\text { migration and invasion in vitro }\end{array}$ & MMP26 $\downarrow$ & Yes (Mouse) & Chen et al., 2016 \\
\hline Hsa-miR-29c & $\begin{array}{l}\text { Overexpression of miR-29c in HEECs impairs adhesion } \\
\text { in vitro }\end{array}$ & COL4A1 $\downarrow$ & Not available & Griffiths et al., 2019 \\
\hline Hsa-miR-590-3p & $\begin{array}{l}\text { Overexpression of miR-590-3p in HEECs in vitro impairs } \\
\text { adhesion }\end{array}$ & $\mathrm{N} / \mathrm{A}$ & Not available & Takamura et al., 2019 \\
\hline Hsa-miR-140 & $\begin{array}{l}\text { Overexpression of miR-140 in RL95-2 endometrial epithelial } \\
\text { cells impairs adhesion and spheroid outgrowth in vitro }\end{array}$ & $\mathrm{N} / \mathrm{A}$ & Yes (Rat) & Sirohi et al., 2018 \\
\hline
\end{tabular}

provide an optimal environment to promote receptivity and implantation (Farrer-Brown et al., 1970). Endometrial cells may secrete/transport a number of miRs to the tissue site of action by way of the blood, and studies suggest that endometrial expression levels of at least some miRs are reflected in the blood (Kresowik et al., 2014; Di Pietro et al., 2018). Circulating miRs in the blood may be able to predict endometrial receptivity and implantation. A previous study used whole blood and paired mid-secretory phase endometrial tissue to determine whether circulating miRs could distinguish fertile from recurrent implantation failure patients (Rekker et al., 2018). miR-30a-5p was identified as differentially expressed in whole blood between the two groups; however, this difference was not reflected in the paired endometrial tissue (Rekker et al., 2018). One possible explanation is that blood cells express miRs (Jickling et al., 2014), which may mask endometrial tissue-secreted miRs. Recent work using paired serum and mid-secretory phase endometrium investigated five miRs and identified a positive correlation of hsa-miR-31 expression levels between serum and endometrial tissue (Kresowik et al., 2014). Alternatively, extracellular miR expression levels do not necessarily reflect cellular expression levels. Whether miR biomarkers have critical functions in endometrial receptivity also needs to be determined experimentally, as differentially expressed circulating miRs between women with normal fertility and infertility may not all have functional relevance in receptivity or implantation. Functional studies of the identified circulating and cellular miRs in receptivity and implantation models could provide evidence to support their potential application as biomarkers and treatment targets. For example, hsa-miR-200c expression is increased in the serum of infertility and abortion patients, compared to healthy women (Zheng et al., 2017). Functional analysis using both human endometrial cell lines and a mouse model demonstrates that hsa-miR-200c overexpression impairs endometrial cell receptivity in both species (Zheng et al., 2017).

The obvious challenge to predict implantation outcome using miRs in the blood will be to distinguish the endometrial secreted miRs from miRs secreted by other tissues. Of note, the process of embryo implantation somewhat resembles that of cancer cell metastasis. Both processes share some of the cellular mechanisms in cell adhesion, invasion, and angiogenesis (Murray and Lessey, 1999). miRs such as hsa-miR-29c (Griffiths et al., 2019) and hsa-miR-125b (Chen et al., 2016) that are dysregulated in the endometrium from infertile women are also associated with gastric and endometrial cancers (Shang et al., 2012; Wang et al., 2019). Cancer cells releasing miRs into the blood may confound the detection of miRs secreted by the endometrium. In this regard, an important feature of the endometrium is that it regenerates itself at each menstrual cycle. The endometrium is only receptive to an implanting embryo within a very short window in the mid-secretory phase (Ashary et al., 2018). Such a functional switch is mediated by coordinated changes of miR expression (Vilella et al., 2015). These phase-dependent changes, in turn, may endow endometrial-secreted miRs with unique cycle-dependent expression fingerprints that can be used to distinguish from the background of other potential 
tissue-secreted miRs. This theory is evidenced by a previous study comparing miR expression between the proliferative phase and mid-secretory phase in paired serum and endometrial tissue from fertile women. hsa-miR-31 has been identified as a potential biomarker that is elevated in both serum and endometrium in the mid-secretory phase, compared to the proliferative phase (Kresowik et al., 2014). It is also essential to investigate appropriate controls from different pathologies as comparative groups. The predictive application of blood miRs on implantation will likely be based on the multiple measurements of $\mathrm{miR}$ expression at different phases within a menstrual cycle.

Another challenge of using miR levels in the blood for biomarker purposes has been identified in cancer diagnosis. A previous study selected 79 solid cancer-circulating miR biomarkers and determined their expression levels in blood cells. Forty-six of the 79 miRs were highly expressed in the blood cells (Pritchard et al., 2012). Plasma isolated from the blood with different blood cell counts or hemolysis impacted the expression levels of select miRs (Pritchard et al., 2012). Inconsistency has also been observed between plasma and serum levels of miR between pregnant and non-pregnant patient groups after embryo transfer (Yang et al., 2018). To improve the accuracy of prediction, a panel of miRs is required, as has been proposed for cancer diagnosis (Madhavan et al., 2013). To achieve this, an investigation of $\mathrm{miR}$ levels from large cohorts of women with different etiologies of infertility and other pathologies is required for their potential use as biomarkers. We have previously identified a dysregulation of miR-processing machinery in the endometrium of a cohort of infertile patients, which would have an overall impact on $\mathrm{miR}$ secretion due to compromised miR processing within the cell (Loke et al., 2019). The miR secretion in this cohort may be different compared to other infertile cohorts caused by different etiologies. Identifying which miRs are responsible for ensuring endometrial receptivity is also required to determine whether the biomarkers may also be useful as treatment targets of dysregulated endometrial receptivity.

\section{UTERINE FLUID}

The uterine fluid is secreted by the human endometrium as an indirect approach to communicate with an embryo for the preparation of implantation. Compared to other body fluids, uterine fluid is a more "local" secretion and, thus, may provide direct information when assessing biomarkers for implantation. Detailed compositional analysis has revealed that uterine fluid contains miRs and proteins with changed profiles across the menstrual cycle (Scotchie et al., 2009; Ng et al., 2013). Functional analysis has proven that endometrial cells secreted miRs, such as hsa-miR-30d, that are taken up by the embryo via the trophectoderm and regulate adhesion in vitro (Vilella et al., 2015). Further investigation demonstrates that secreted miRs in the uterine fluid target an extensive of implantation-related genes ( $\mathrm{Ng}$ et al., 2013). Of note, the miRs in the uterine fluid can be sourced from different endometrial cells and the blood. This can be determined via in situ hybridization on endometrial sections, like what has been done for protein via immunostaining (Hannan et al., 2010). Uterine fluid can be collected via either aspiration or lavage without compromising implantation (Hannan et al., 2012). To the best of our knowledge, however, most currently available studies on uterine fluid have focused on comparing the proteins between fertile and infertile patients (Hannan et al., 2010; Salamonsen et al., 2013). There are presently limited studies investigating the potential of using miRs in the uterine fluid as a diagnostic approach for implantation.

\section{OVERALL CHALLENGES OF USING SECRETED MIRS TO PREDICT IMPLANTATION}

Although miRs are highly desirable as non-invasive biomarkers to predict implantation, this field of research is somewhat confounded by a general inconsistency of miR expression levels across different studies. It has been identified that a number of factors including RNA isolation and detection systems can contribute to this inconsistency. Recently published work from one laboratory, which used different commercial kits to isolate RNA, demonstrated that the recovery of RNA was variable between the commercial kits (El-Khoury et al., 2016; Wright et al., 2020). In addition, the selection of endogenous controls to normalize the target $\mathrm{miR}$ expression levels directly affects the results, and such importance has been neglected by some studies. For miR normalization, an ideal endogenous control should be stably expressed in the body fluid with minimal biological variation, and the expression should not change with different implantation outcomes. It is known that in body fluids, the expression of some cellular endogenous controls may vary between different samples bringing deviation in normalization. It is an essential first step to compare the expression variability of a number of endogenous control candidates in a given body fluid system and confirm their stability. This has not been conducted in some studies and may have contributed to the variability of miR expression. A workflow has been proposed to identify the best normalization control (Schwarzenbach et al., 2015). All these steps introducing impact factors require standardization before a solid conclusion can be drawn.

Adding to this challenge is the observation that inherent differences between women, together with different IVF protocols, may lead to differential expression patterns of miR in the human endometrium. A microarray study has identified that luteal support following controlled ovarian stimulation has a profound influence on the miR profile in the endometrium (Zhao et al., 2012). Specifically, progesterone supplementation is associated with a significant increase in miR expression in the endometrium compared to a no steroid supplementation group following controlled ovarian stimulation (Zhao et al., 2012). The findings are in accordance with a previous report identifying differential expression patterns of $\mathrm{miR}$ between natural and stimulated IVF cycles (Sha et al., 2011). In addition, patients receiving the same IVF treatment who have different serum progesterone levels have been identified to have different miR expression patterns in the endometrial tissue collected 6 days 
after oocyte retrieval ( $\mathrm{Li}$ et al., 2011). Microarray analysis of the endometrium identified four miRs (hsa-miR-451, hsa-miR424, hsa-miR-125b, and hsa-miR-30b) that were decreased in the high serum progesterone group (Li et al., 2011). The effects of controlled ovarian stimulation and luteal phase support need to be considered when comparing data from different studies.

\section{CONCLUSION}

Measurement of miRs in the samples that can be collected without compromising embryo transfer in the same menstrual cycle opens new perspectives for the diagnosis of embryo implantation potential. Unfortunately, our understanding of the mechanisms of how miR dysregulation impacts implantation and how this accordingly affects miR secretion remains far from complete. Interpretation of research findings is confounded by unstandardized assessment of miRs in a given body fluid. Resolving these questions would have a major impact on biomarker development and clinical practice for reproductive clinicians and scientists. This includes optimizing the selection of embryos for transfer during IVF, improvement of implantation success rates, and the minimization of multiple pregnancies. It is likely that a combination of samples that can be collected either non-invasively or relatively non-invasively, as summarized in this review, will be useful to assess implantation potential at different

\section{REFERENCES}

Abd El Naby, W. S. H. (2012). Expression analysis of regulatory MicroRNA in bovine cumulus oocyte complex and preimplantation embryos. Zygote 21, 31-51. doi: 10.1017/s0967199411000566

Abu-Halima, M., Khaizaran, Z. A., Ayesh, B. M., Fischer, U., Khaizaran, S. A., AlBattah, F., et al. (2020). MicroRNAs in combined spent culture media and sperm are associated with embryo quality and pregnancy outcome. Fertil. Steril. 113, 970.e2-980.e2

Ashary, N., Tiwari, A., and Modi, D. (2018). Embryo implantation: war in times of love. Endocrinology 159, 1188-1198. doi: 10.1210/en.2017-03082

Bai, R., Latifi, Z., Kusama, K., Nakamura, K., Shimada, M., and Imakawa, K. (2018). Induction of immune-related gene expression by seminal exosomes in the porcine endometrium. Biochem. Biophys. Res. Commun. 495, 1094-1101. doi: 10.1016/j.bbrc.2017.11.100

Balaguer, N., Moreno, I., Herrero, M., Gonzáléz-Monfort, M., Vilella, F., and Simón, C. (2019). MicroRNA-30d deficiency during preconception affects endometrial receptivity by decreasing implantation rates and impairing fetal growth. Am. J. Obstet. Gynecol. 221, 46.e1-46.e16.

Belandres, D., Shamonki, M., and Arrach, N. (2019). Current status of spent embryo media research for preimplantation genetic testing. J. Assist. Reprod. Genet. 36, 819-826. doi: 10.1007/s10815-019-01437-6

Boomsma, C., Kavelaars, A., Eijkemans, M., Lentjes, E., Fauser, B., Heijnen, C., et al. (2009). Endometrial secretion analysis identifies a cytokine profile predictive of pregnancy in IVF. Hum. Reprod. 24, 1427-1435. doi: 10.1093/humrep/dep011

Borges, E. Jr., Setti, A. S., Braga, D. P., Geraldo, M. V., Figueira, R. D. C. S., and Iaconelli, A. Jr. (2016). miR-142-3p as a biomarker of blastocyst implantation failure-A pilot study. JBRA Assist. Reprod. 20, 200-205. doi: 10.5935/15180557.20160039

Bushati, N., and Cohen, S. M. (2007). microRNA functions. Annu. Rev. Cell Dev. Biol. 23, 175-205.

Cai, J. L., Liu, L. L., Hu, Y., Jiang, X. M., Qiu, H. L., Sha, A.-G., et al. (2016). Polychlorinated biphenyls impair endometrial receptivity in vitro via regulating mir-30d expression and epithelial mesenchymal transition. Toxicology 365, 25-34. doi: 10.1016/j.tox.2016.07.017 stages of conceptus establishment and development. This relies on research to find $\mathrm{miR}$ biomarkers related to implantation regulation and the development of new technologies to improve miR detection. A few microfluidic devices have been developed recently with a larger capacity to include more miRs and reduce analysis time. Improved diagnosis of embryo implantation could have a profound effect on psychological and financial well-being on women and couples undergoing IVF treatment.

\section{AUTHOR CONTRIBUTIONS}

Both authors made substantial contributions to the conception of this review and the critical appraisal of the literature summarized herein, wrote the manuscript, and approved the final version of this article before submission.

\section{FUNDING}

This work was supported by a project grant (APP1120689) and a senior research fellowship (\#550905) from the National Health and Medical Research Council (NHMRC) of Australia to ED. WZ was supported by an Early Career Researcher Grant and a Department of Obstetrics and Gynaecology Innovation Grant (The University of Melbourne).

Capalbo, A., Ubaldi, F. M., Cimadomo, D., Noli, L., Khalaf, Y., Farcomeni, A., et al. (2016). MicroRNAs in spent blastocyst culture medium are derived from trophectoderm cells and can be explored for human embryo reproductive competence assessment. Fertil. Steril. 105, 225-235. doi: 10.1016/j.fertnstert. 2015.09.014

Chachamovich, J. R., Chachamovich, E., Ezer, H., Fleck, M. P., Knauth, D., and Passos, E. P. (2010). Investigating quality of life and health-related quality of life in infertility: a systematic review. J. Psychosom. Obstet. Gynaecol. 31, 101-110. doi: 10.3109/0167482x.2010.481337

Chen, C., Zhao, Y., Yu, Y., Li, R., and Qiao, J. (2016). MiR-125b regulates endometrial receptivity by targeting MMP26 in women undergoing IVF-ET with elevated progesterone on HCG priming day. Sci. Rep. 6:25302.

Chu, B., Zhong, L., Dou, S., Wang, J., Li, J., Wang, M., et al. (2015). miRNA-181 regulates embryo implantation in mice through targeting leukemia inhibitory factor. J. Mol. Cell Biol. 7, 12-22. doi: 10.1093/jmcb/mjv006

Conine, C. C., Sun, F., Song, L., Rivera-Pérez, J. A., and Rando, O. J. (2018). Small RNAs gained during epididymal transit of sperm are essential for embryonic development in mice. Dev. Cell 46, 470-480. doi: 10.1016/j.devcel.2018.06.024

Conine, C. C., Sun, F., Song, L., Rivera-Pérez, J. A., and Rando, O. J. (2019). MicroRNAs absent in caput sperm are required for normal embryonic development. Dev. Cell 50, 7-8. doi: 10.1016/j.devcel.2019. 06.007

Craciunas, L., Gallos, I., Chu, J., Bourne, T., Quenby, S., Brosens, J. J., et al. (2019). Conventional and modern markers of endometrial receptivity: a systematic review and meta-analysis. Hum. Reprod. Upd. 25, 202-223. doi: 10.1093/ humupd/dmy044

Cui, W. (2010). Mother or nothing: the agony of infertility. Bull. World Health Organ. 88, 881-882. doi: 10.2471/blt.10.011210

Cuman, C., Menkhorst, E., Rombauts, L., Holden, S., Webster, D., Bilandzic, M., et al. (2013). Preimplantation human blastocysts release factors that differentially alter human endometrial epithelial cell adhesion and gene expression relative to IVF success. Hum. Reprod. 28, 1161-1171. doi: 10.1093/ humrep/det058

Cuman, C., Van Sinderen, M., Gantier, M. P., Rainczuk, K., Sorby, K., Rombauts, L., et al. (2015). Human blastocyst secreted microRNA regulate endometrial 
epithelial cell adhesion. eBio Med. 2, 1528-1535. doi: 10.1016/j.ebiom.2015. 09.003

Devjak, R., Papler, T. B., Verdenik, I., Tacer, K. F., and Bokal, E. V. (2016). Embryo quality predictive models based on cumulus cells gene expression. Balkan J. Med. Genet. 19, 5-12. doi: 10.1515/bjmg-2016-0001

Di Pietro, C., Caruso, S., Battaglia, R., Iraci Sareri, M., La Ferlita, A., Strino, F., et al. (2018). MiR-27a-3p and miR-124-3p, upregulated in endometrium and serum from women affected by Chronic Endometritis, are new potential molecular markers of endometrial receptivity. Am. J. Reprod. Immunol. 80:e12858. doi: 10.1111/aji.12858

Díaz-Gimeno, P., Horcajadas, J. A., Martínez-Conejero, J. A., Esteban, F. J., Alamá, P., Pellicer, A., et al. (2011). A genomic diagnostic tool for human endometrial receptivity based on the transcriptomic signature. Fertil. Steril. 95, 50-60. doi: 10.1016/j.fertnstert.2010.04.063

El-Khoury, V., Pierson, S., Kaoma, T., Bernardin, F., and Berchem, G. (2016). Assessing cellular and circulating miRNA recovery: the impact of the RNA isolation method and the quantity of input material. Sci. Rep. 6:19529.

Farrer-Brown, G., Beilby, J., and Tarbit, M. (1970). The blood supply of the uterus: 1. Arterial vasculature. J. Obstet. Gynaecol. Br. Common. 77, 673-681. doi: 10.1111/j.1471-0528.1970.tb03592.x

Fu, J., Qu, R. G., Zhang, Y. J., Gu, R. H., Li, X., Sun, Y. J., et al. (2018). Screening of miRNAs in human follicular fluid reveals an inverse relationship between microRNA-663b expression and blastocyst formation. Reprod. Biomed. Online 37, 25-32. doi: 10.1016/j.rbmo.2018.03.021

Gardner, D. K., and Balaban, B. (2016). Assessment of human embryo development using morphological criteria in an era of time-lapse, algorithms and 'OMICS': is looking good still important? Mol. Hum. Reprod. 22, 704-718. doi: 10.1093/ molehr/gaw057

Gardner, D. K., Lane, M., Stevens, J., Schlenker, T., and Schoolcraft, W. B. (2000). Blastocyst score affects implantation and pregnancy outcome: towards a single blastocyst transfer. Fertil. Steril. 73, 1155-1158. doi: 10.1016/s0015-0282(00) 00518-5

Gasca, S., Pellestor, F., Assou, S., Loup, V., Anahory, T., Dechaud, H., et al. (2007). Identifying new human oocyte marker genes: a microarray approach. Reprod. Biomed. Online 14, 175-183. doi: 10.1016/s1472-6483(10)60785-7

Giorgetti, C., Terriou, P., Auquier, P., Hans, E., Spach, J. L., Salzmann, J., et al. (1995). Implantation: embryo score to predict implantation after in-vitro fertilization: based on 957 single embryo transfers. Hum. Reprod. 10, $2427-$ 2431. doi: 10.1093/oxfordjournals.humrep.a136312

Griffiths, M., Van Sinderen, M., Rainczuk, K., and Dimitriadis, E. (2019). miR-29c overexpression and COL4A1 downregulation in infertile human endometrium reduces endometrial epithelial cell adhesive capacity in vitro implying roles in receptivity. Sci. Rep. 9:8644.

Hamel, M., Dufort, I., Robert, C., Gravel, C., Leveille, M. C., Leader, A., et al. (2008). Identification of differentially expressed markers in human follicular cells associated with competent oocytes. Hum. Reprod. 23, 1118-1127. doi: 10.1093/humrep/den048

Hannan, N. J., Nie, G., Rainzcuk, A., Rombauts, L., and Salamonsen, L. A. (2012). Uterine lavage or aspirate: which view of the intrauterine environment? Reprod. Sci. 19, 1125-1132. doi: 10.1177/1933719112443879

Hannan, N. J., Stephens, A. N., Rainczuk, A., Hincks, C., Rombauts, L. J., and Salamonsen, L. A. (2010). 2D-DiGE analysis of the human endometrial secretome reveals differences between receptive and nonreceptive states in fertile and infertile women. J. Proteome Res. 9, 6256-6264. doi: 10.1021/ pr1004828

Huang, Z., and Wells, D. (2010). The human oocyte and cumulus cells relationship: new insights from the cumulus cell transcriptome. Mol. Hum. Reprod. 16, 715-725. doi: 10.1093/molehr/gaq031

Jickling, G. C., Ander, B. P., Zhan, X., Noblett, D., Stamova, B., and Liu, D. (2014). microRNA expression in peripheral blood cells following acute ischemic stroke and their predicted gene targets. PLoS One 9:e99283. doi: 10.1371/journal.pone. 0099283

Kang, Y. J., Lees, M., Matthews, L. C., Kimber, S. J., Forbes, K., and Aplin, J. D. (2015). miR-145 suppresses embryo-epithelial juxtacrine communication at implantation by modulating maternal IGF1R. J. Cell Sci. 128, 804-814. doi: $10.1242 /$ jcs. 164004

Kottawatta, K. S., So, K. H., Kodithuwakku, S. P., Ng, E. H., Yeung, W. S., and Lee, K. F. (2015). MicroRNA-212 regulates the expression of olfactomedin 1 and
C-terminal binding protein 1 in human endometrial epithelial cells to enhance spheroid attachment in vitro. Biol. Reprod. 93:109.

Kresowik, J. D., Devor, E. J., Van Voorhis, B. J., and Leslie, K. K. (2014). MicroRNA31 is significantly elevated in both human endometrium and serum during the window of implantation: a potential biomarker for optimum receptivity. Biol. Reprod. 91:17.

Kropp, J., Salih, S. M., and Khatib, H. (2014). Expression of microRNAs in bovine and human pre-implantation embryo culture media. Front Genet 5:91. doi: 10.3389/fgene.2014.00091

Li, R., Qiao, J., Wang, L., Li, L., Zhen, X., Liu, P., et al. (2011). MicroRNA array and microarray evaluation of endometrial receptivity in patients with high serum progesterone levels on the day of hCG administration. Reprod. Biol. Endocrinol. 9:29. doi: 10.1186/1477-7827-9-29

Li, T., Greenblatt, E., and Chan, C. (2019). Isolation and profiling of extracellular vesicles in uterine fluid to determine novel markers of endometrial receptivity. Fertil. Steril. 112:e314. doi: 10.1016/j.fertnstert.2019.07.911

Loke, H., Rainczuk, K., and Dimitriadis, E. (2019). MicroRNA biogenesis machinery is dysregulated in the endometrium of infertile women suggesting a role in receptivity and infertility. J. Histochem. Cytochem. 67, 589-599. doi: $10.1369 / 0022155419854064$

Machtinger, R., Laurent, L. C., and Baccarelli, A. A. (2016). Extracellular vesicles: roles in gamete maturation, fertilization and embryo implantation. Hum. Reprod. Upd. 22, 182-193.

Machtinger, R., Rodosthenous, R. S., Adir, M., Mansour, A., Racowsky, C., Baccarelli, A. A., et al. (2017). Extracellular microRNAs in follicular fluid and their potential association with oocyte fertilization and embryo quality: an exploratory study. J. Assist. Reprod. Genet. 34, 525-533. doi: 10.1007/s10815017-0876-8

Madhavan, D., Cuk, K., Burwinkel, B., and Yang, R. (2013). Cancer diagnosis and prognosis decoded by blood-based circulating microRNA signatures. Front. Genet. 4:116. doi: 10.3389/fgene.2013.00116

McCallie, B., Schoolcraft, W. B., and Katz-Jaffe, M. G. (2010). Aberration of blastocyst microRNA expression is associated with human infertility. Fertil. Steril. 93, 2374-2382. doi: 10.1016/j.fertnstert.2009.01.069

Mokánszki, A., Molnár, Z., Varga Tóthné, E., Bodnár, B., Jakab, A., Bálint, B. L., et al. (2019). Altered microRNAs expression levels of sperm and seminal plasma in patients with infertile ejaculates compared with normozoospermic males. Hum. Fertil. [Epub ahead of print].

Murray, M. J., and Lessey, B. A. (1999). Embryo Implantation, and Tumor Metastasis: Common Pathways of Invasion, and Angiogenesis, Semin Reprod Endocrinol. New York, NY: Thieme Medical Publishers, 275-290.

Ng, Y. H., Rome, S., Jalabert, A., Forterre, A., Singh, H., Hincks, C. L., et al. (2013). Endometrial exosomes/microvesicles in the uterine microenvironment: a new paradigm for embryo-endometrial cross talk at implantation. PLoS One 8:e58502. doi: 10.1371/journal.pone.0058502

Nixon, B., Stanger, S. J., Mihalas, B. P., Reilly, J. N., Anderson, A. L., Tyagi, S., et al. (2015). The microRNA signature of mouse spermatozoa is substantially modified during epididymal maturation. Biol. Reprod. 93:91.

Norwitz, E. R., Schust, D. J., and Fisher, S. J. (2001). Implantation and the survival of early pregnancy. N. Engl. J. Med. 345, 1400-1408. doi: 10.1056/nejmra000763

Patrizio, P., Fragouli, E., Bianchi, V., Borini, A., and Wells, D. (2007). Molecular methods for selection of the ideal oocyte. Reprod. Biomed. Online 15, 346-353. doi: 10.1016/s1472-6483(10)60349-5

Paul, A. B., Sadek, S. T., and Mahesan, A. M. (2019). The role of microRNAs in human embryo implantation: a review. J. Assist. Reprod. Genet. 36, 179-187. doi: 10.1007/s10815-018-1326-y

Pritchard, C. C., Kroh, E., Wood, B., Arroyo, J. D., Dougherty, K. J., Miyaji, M. M., et al. (2012). Blood cell origin of circulating microRNAs: a cautionary note for cancer biomarker studies. Cancer Prev. Res. 5, 492-497. doi: 10.1158/19406207.capr-11-0370

Ralt, D., Goldenberg, M., Fetterolf, P., Thompson, D., Dor, J., Mashiach, S., et al. (1991). Sperm attraction to a follicular factor (s) correlates with human egg fertilizability. Proc. Natl. Acad. Sci. U.S.A. 88, 2840-2844. doi: 10.1073/pnas. 88.7.2840

Reilly, J. N., McLaughlin, E. A., Stanger, S. J., Anderson, A. L., Hutcheon, K., Church, K., et al. (2016). Characterisation of mouse epididymosomes reveals a complex profile of microRNAs and a potential mechanism for modification of the sperm epigenome. Sci. Rep. 6, 1-15. 
Rekker, K., Altmäe, S., Suhorutshenko, M., Peters, M., Martinez-Blanch, J. F., Codoñer, F. M., et al. (2018). A two-cohort RNA-seq study reveals changes in endometrial and blood miRNome in fertile and infertile women. Genes 9:574. doi: 10.3390/genes9120574

Robertson, S. A., and Sharkey, D. J. (2016). Seminal fluid and fertility in women. Fertil. Steril. 106, 511-519. doi: 10.1016/j.fertnstert.2016.07.1101

Rolland, A. D., Lavigne, R., Dauly, C., Calvel, P., Kervarrec, C., Freour, T., et al. (2013). Identification of genital tract markers in the human seminal plasma using an integrative genomics approach. Hum. Reprod. 28, 199-209. doi: 10. 1093/humrep/des360

Rosenbluth, E. M., Shelton, D. N., Wells, L. M., Sparks, A. E., and Van Voorhis, B. J. (2014). Human embryos secrete microRNAs into culture media-a potential biomarker for implantation. Fertil. Steril. 101, 1493-1500. doi: 10.1016/j. fertnstert.2014.01.058

Salamonsen, L. A., Edgell, T., Rombauts, L. J., Stephens, A. N., Robertson, D. M., Rainczuk, A., et al. (2013). Proteomics of the human endometrium and uterine fluid: a pathway to biomarker discovery. Fertil. Steril. 99, 1086-1092. doi: 10.1016/j.fertnstert.2012.09.013

Scalici, E., Traver, S., Mullet, T., Molinari, N., Ferrieres, A., Brunet, C., et al. (2016). Circulating microRNAs in follicular fluid, powerful tools to explore in vitro fertilization process. Sci. Rep. 6:24976.

Schwarzenbach, H., Da Silva, A. M., Calin, G., and Pantel, K. (2015). Data normalization strategies for microRNA quantification. Clin. Chem. 61, 13331342. doi: 10.1373/clinchem.2015.239459

Schwarzenbach, H., Nishida, N., Calin, G. A., and Pantel, K. (2014). Clinical relevance of circulating cell-free microRNAs in cancer. Nat. Rev. Clin. Oncol. 11, 145-156. doi: 10.1038/nrclinonc.2014.5

Scotchie, J. G., Fritz, M. A., Mocanu, M., Lessey, B. A., and Young, S. L. (2009). Proteomic analysis of the luteal endometrial secretome. Reprod. Sci. 16, 883893. doi: $10.1177 / 1933719109337165$

Sha, A. G., Liu, J. L., Jiang, X. M., Ren, J. Z., Ma, C. H., Lei, W., et al. (2011). Genome-wide identification of micro-ribonucleic acids associated with human endometrial receptivity in natural and stimulated cycles by deep sequencing. Fertil. Steril. 96, 150-155. doi: 10.1016/j.fertnstert.2011.04.072

Shang, C., Lu, Y. M., and Meng, L. R. (2012). MicroRNA-125b down-regulation mediates endometrial cancer invasion by targeting ERBB2. Med. Sci. Monit. 18, BR149-BR155.

Sharma, U., Conine, C. C., Shea, J. M., Boskovic, A., Derr, A. G., Bing, X. Y., et al. (2016). Biogenesis and function of tRNA fragments during sperm maturation and fertilization in mammals. Science 351, 391-396. doi: 10.1126/science. aad 6780

Sharma, U., Sun, F., Conine, C. C., Reichholf, B., Kukreja, S., Herzog, V. A., et al. (2018). Small RNAs are trafficked from the epididymis to developing mammalian sperm. Dev. Cell 46, 481-494. doi: 10.1016/j.devcel.2018.06.023

Sirohi, V. K., Gupta, K., Kumar, R., Shukla, V., and Dwivedi, A. (2018). Selective estrogen receptor modulator Ormeloxifene suppresses embryo implantation via inducing miR-140 and targeting insulin-like growth factor 1 receptor in rat uterus. J. Steroid Biochem. Mol. Biol. 178, 272-282. doi: 10.1016/j.jsbmb.2018. 01.006

Takamura, M., Zhou, W., Rombauts, L., and Dimitriadis, E. (2019). The long noncoding RNA PTENP1 regulates human endometrial epithelial adhesive capacity in vitro: implications in infertility. Biol. Reprod. 102, 53-62. doi: 10.1093/biolre/ioz173

Tompkins, A. J., Chatterjee, D., Maddox, M., Wang, J., Arciero, E., Camussi, G., et al. (2015). The emergence of extracellular vesicles in urology: fertility, cancer, biomarkers and targeted pharmacotherapy. J. Extracell. Vesicles 4:23815. doi: $10.3402 /$ jev.v4.23815

Trigg, N. A., Eamens, A. L., and Nixon, B. (2019). The contribution of epididymosomes to the sperm small RNA profile. Reproduction 157, R209R223.

Vilella, F., Moreno-Moya, J. M., Balaguer, N., Grasso, A., Herrero, M., Martínez, S., et al. (2015). Hsa-miR-30d, secreted by the human endometrium, is taken up by the pre-implantation embryo and might modify its transcriptome. Development 142, 3210-3221. doi: 10.1242/dev.124289
Vojtech, L., Woo, S., Hughes, S., Levy, C., Ballweber, L., Sauteraud, R. P., et al. (2014). Exosomes in human semen carry a distinctive repertoire of small non-coding RNAs with potential regulatory functions. Nucleic Acids Res. 42, 7290-7304. doi: 10.1093/nar/gku347

Wang, L., Yu, T., Li, W., Li, M., Zuo, Q., Zou, Q., et al. (2019). The miR-29c-KIAA1199 axis regulates gastric cancer migration by binding with WBP11 and PTP4A3. Oncogene 38, 3134-3150. doi: 10.1038/s41388-0180642-0

Wilcox, A. J., Weinberg, C. R., O'Connor, J. F., Baird, D. D., Schlatterer, J. P., Canfield, R. E., et al. (1988). Incidence of early loss of pregnancy. N. Engl. J. Med. 319, 189-194.

Winship, A., Ton, A., Van Sinderen, M., Menkhorst, E., Rainczuk, K., Griffiths, M., et al. (2018). Mouse double minute homologue 2 (MDM2) downregulation by miR-661 impairs human endometrial epithelial cell adhesive capacity. Reprod. Fertil. Dev. 30, 477-486. doi: 10.1071/rd17095

Wright, K., de Silva, K., Purdie, A. C., and Plain, K. M. (2020). Comparison of methods for miRNA isolation and quantification from ovine plasma. Sci. Rep. 10:825.

Xu, B., Zhang, Y. W., Tong, X. H., and Liu, Y. S. (2015). Characterization of microRNA profile in human cumulus granulosa cells: identification of microRNAs that regulate Notch signaling and are associated with PCOS. Mol. Cell. Endocrinol. 404, 26-36. doi: 10.1016/j.mce.2015.01.030

Xu, H., Wang, X., Wang, Z., Li, J., Xu, Z., Miao, M., et al. (2020). MicroRNA expression profile analysis in sperm reveals hsa-mir-191 as an auspicious omen of in vitro fertilization. BMC Genomics 21:165. doi: 10.1186/s12864-0206570-8

Yang, Q., Gu, W. W., Gu, Y., Yan, N. N., Mao, Y. Y., Zhen, X. X., et al. (2018). Association of the peripheral blood levels of circulating microRNAs with both recurrent miscarriage and the outcomes of embryo transfer in an in vitro fertilization process. J. Transl. Med. 16:186.

Yuan, S., Schuster, A., Tang, C., Yu, T., Ortogero, N., Bao, J., et al. (2016). Sperm-borne miRNAs and endo-siRNAs are important for fertilization and preimplantation embryonic development. Development 143, 635-647. doi: 10 . 1242/dev.131755

Zhang, Q., Zhang, H., Jiang, Y., Xue, B., Diao, Z., Ding, L., et al. (2015). MicroRNA$181 \mathrm{a}$ is involved in the regulation of human endometrial stromal cell decidualization by inhibiting Krüppel-like factor 12. Reprod. Biol. Endocrinol. 13:23.

Zhao, Y., Zacur, H., Cheadle, C., Ning, N., Fan, J., and Vlahos, N. F. (2012). Effect of luteal-phase support on endometrial microRNA expression following controlled ovarian stimulation. Reprod. Biol. Endocrinol. 10:72. doi: 10.1186/ 1477-7827-10-72

Zheng, Q., Zhang, D., Yang, Y. U., Cui, X., Sun, J., Liang, C., et al. (2017). MicroRNA-200c impairs uterine receptivity formation by targeting FUT4 and $\alpha$ 1, 3-fucosylation. Cell Death. Differ. 24, 2161-2172. doi: 10.1038/cdd.2017.136 Zhou, W., De Iuliis, G. N., Dun, M. D., and Nixon, B. (2018). Characteristics of the epididymal luminal environment responsible for sperm maturation and storage. Front. Endocrinol. 9:59. doi: 10.3389/fendo.2018. 00059

Zhou, W., Stanger, S. J., Anderson, A. L., Bernstein, I. R., De Iuliis, G. N., McCluskey, A., et al. (2019). Mechanisms of tethering and cargo transfer during epididymosome-sperm interactions. BMC Biol. 17:35. doi: 10.1186/s12915019-0653-5

Conflict of Interest: The authors declare that the research was conducted in the absence of any commercial or financial relationships that could be construed as a potential conflict of interest.

Copyright (C) 2020 Zhou and Dimitriadis. This is an open-access article distributed under the terms of the Creative Commons Attribution License (CC BY). The use, distribution or reproduction in other forums is permitted, provided the original author(s) and the copyright owner(s) are credited and that the original publication in this journal is cited, in accordance with accepted academic practice. No use, distribution or reproduction is permitted which does not comply with these terms. 